\title{
Evidence that Synchrotron Emission from Nonthermal Electrons Produces the Increasing Submillimeter Spectral Component in Solar Flares
}

\author{
Adriana V.R. Silva • G.H. Share • R.J. Murphy • \\ J.E.R. Costa • C.G. Giménez de Castro · J.-P. Raulin • \\ P. Kaufmann
}

Received: 25 May 2007 / Accepted: 6 September 2007 / Published online: 8 November 2007

(C) Springer Science+Business Media B.V. 2007

\begin{abstract}
We investigate the origin of the increasing spectra observed at submillimeter wavelengths detected in the flare on 2 November 2003 starting at 17:17 UT. This flare, classified as an X8.3 and 2B event, was simultaneously detected by RHESSI and the Solar Submillimeter Telescope (SST) at 212 and $405 \mathrm{GHz}$. Comparison of the time profiles at various wavelengths shows that the submillimeter emission resembles that of the high-energy $\mathrm{X}$ rays observed by RHESSI whereas the microwaves observed by the Owens Valley Solar Array (OVSA) resemble that of $\sim 50 \mathrm{keV} \mathrm{X}$ rays. Moreover, the centroid position of the submillimeter radiation is seen to originate within the same flaring loops of the ultraviolet and X-ray sources. Nevertheless, the submillimeter spectra are distinct from the usual microwave spectra, appearing to be a distinct spectral component with peak frequency in the $\mathrm{THz}$ range. Three possibilities to explain this increasing radio spectra are discussed: (1) gyrosynchrotron radiation from accelerated electrons, (2) bremsstrahlung from thermal electrons, and (3) gyrosynchrotron emission from the positrons produced by pion or radioactive decay after nuclear interactions. The latter possibility is ruled out on the grounds that to explain the submillimeter observations requires 3000 to $2 \times 10^{5}$ more positrons than what is inferred from X-ray and $\gamma$-ray observations. It is possible to model the emission as thermal; however, such sources would produce too much flux in the ultraviolet and soft X-ray
\end{abstract}

A.V.R. Silva $(\bowtie)$ · C.G. Giménez de Castro · J.-P. Raulin · P. Kaufmann

CRAAM, Universidade Presbiteriana Mackenzie, Rua da Consolação, 896, São Paulo, SP 01302, Brazil

e-mail: asilva@ craam.mackenzie.br

G.H. Share

Department of Astronomy, University of Maryland, College Park, MD 20742, USA

G.H. Share · R.J. Murphy

E. O. Hulburt Center for Space Research, Code 7652, Naval Research Laboratory, Washington, DC 20375-5352, USA

J.E.R. Costa

DAS, INPE, São José dos Campos, SP, Brazil

P. Kaufmann

CCS, Universidade Estadual de Campinas, Campinas, SP 013983-970, Brazil 
wavelengths. Nevertheless we are able to explain both spectral components at microwave and submillimeter wavelengths by gyrosynchrotron emission from the same population of accelerated electrons that emit hard $X$ rays and $\gamma$ rays. We find that the same $5 \times 10^{35}$ electrons inferred from RHESSI observations are responsible for the compact submillimeter source ( 0.5 arcsec in radius) in a region of $4500 \mathrm{G}$ low in the atmosphere, and for the traditional microwave spectral component by a more extended source $(50 \mathrm{arcsec})$ in a $480 \mathrm{G}$ magnetic field located higher up in the loops. The extreme values in magnetic field and source size required to account for the submillimeter emission can be relaxed if anisotropy and transport of the electrons are taken into account.

Keywords Sun: solar flares · Sun: synchrotron radiation $\cdot$ Sun: positrons

\section{Introduction}

Since the first detection of solar flares in the submillimeter band above $200 \mathrm{GHz}$ (Trottet et al., 2002) more than 10 additional flares have been observed in this band. Most of these flares exhibit subsecond pulses in the $>200 \mathrm{GHz}$ emission (Raulin et al., 2003). These flare observations revealed a new phenomenon: When simultaneous data at lower radio frequencies are also available, two different types of spectral behavior are observed. Flares have been observed where the $212-\mathrm{GHz}$ observations are consistent with extrapolation of the lower frequency gyrosynchrotron emission from microwaves to submillimeter wavelengths (Trottet et al., 2002; Kaufmann et al., 2002; Raulin et al., 2004; Lüthi, Magun, and Miller, 2004). In contrast (Kaufmann et al., 2004) discovered a new spectral component that increased with frequency above $200 \mathrm{GHz}$ in the 4 November 2003 solar flare. Lüthi, Lüdi, and Magun (2004) identified the same spectral component with a different telescope during the decay phase of the 28 October 2003 flare.

The source of this new spectral component that rises with frequency at submillimeter wavelengths is uncertain. Possible explanations for this high radio frequency spectral component include thermal bremsstrahlung from an optically thick source (Ohki and Hudson, 1975), electron synchrotron emission (Stein and Ney, 1963), synchrotron radiation from positrons emitted in pion or radioactive decay after nuclear interactions (Murphy, Dermer, and Ramaty, 1987; Trottet et al., 2004; Trottet, 2006), and inverse Compton radiation that may explain the rapid pulsations from synchrotron emission (Kaufmann et al., 1986). More recent studies include the production of $\mathrm{THz}$ incoherent synchrotron radiation by an ultrarelativistic beam that undergoes density inhomogeneities giving rise, at the same time, to intense broadband microwave coherent synchrotron radiation by a microbunching instability (Kaufmann and Raulin, 2006). Significant $\mathrm{THz}$ emission may also be produced by plasma waves excited by relativistic electron beams (Sakai et al., 2006) or by proton beams (Sakai and Nagasugi, 2007) at denser regions of the solar atmosphere.

More speculative studies include the possible production of $\mathrm{THz}$ radiation by relativistic electron beams directed into deeper chromosphere-photosphere levels (Sakai et al., 2006) and ultrarelativistic electron beams undergoing density modulations that create a microbunching instability, giving rise to bright and broadband coherent synchrotron observed at microwaves while producing incoherent synchrotron emission in the $\mathrm{THz}$ range, similar to what is observed in laboratory accelerators (Kaufmann and Raulin, 2006).

In this paper we discuss observations of the new submillimeter component by the Solar Submillimeter Telescope (SST, Kaufmann et al., 2001) in the 2 November 2003 flare. This flare was also observed in its entirety by the Ramaty High Energy Solar Spectrometer 
and Imager (RHESSI, Lin et al., 2002). This enables us to determine the spectrum of the flare-accelerated electrons that interact in the solar atmosphere and to determine whether they may be responsible for the new $\mathrm{THz}$ emission through their gyrosynchrotron emission. RHESSI is also able to estimate the flux of positrons released in radioactive decays and to set limits on high-energy positrons from pion decay; we can also determine whether these fluxes can produce the new submillimeter component from their gyrosynchrotron emission. Using properties of the observed 212- and 405-GHz emission we discuss whether it can arise from thermal bremsstrahlung. The observations are described in the next section and Section 3 describes the radio spectrum and its plausible interpretations. The conclusions are presented in Section 4.

\section{Observations}

The flare of 2 November 2003 commenced at 17:07 UT from active region 10486 (S17W63). It was classified as a GOES X8.3 and 2B flare. The flare was detected simultaneously by the SST at 212 and $405 \mathrm{GHz}$, by RHESSI at X- and $\gamma$-ray energies, and by the Owens Valley Solar Array (OVSA) at microwave wavelengths.

\subsection{Radio and X-ray Imaging}

At the time of the flare, the SST was tracking active region 10486 with the multiple beam arrangement shown in Figure 1. The 212-GHz beams indicated by 1 through 4 have FWHP of 4 arcmin; the $405-\mathrm{GHz}$ beam indicated by 5 has the oblong shape illustrated in the figure. (A second $405-\mathrm{GHz}$ beam was not available at the time of the flare.) To obtain the solar flare submillimeter flux density, we first removed the contribution of the Earth's atmosphere to the signal by subtracting the emission in beam 1, pointing 10 arcmin away from the flaring region, from each of the other beams. Emission in beam 1 was scaled such that its maximum and minimum emission well after the flare coincided with those of each of the other beams. These scaling constants were multiplied by the beam 1 emission during the flaring period and this flux density was subtracted from all other beams. Next the signals at both frequencies were temperature calibrated using the quiet Sun scan performed prior to the flare at 17:12:40 UT. The quiet Sun temperatures were assumed to be 5900 and $5100 \mathrm{~K}$ at 212 and $405 \mathrm{GHz}$, respectively (Silva et al., 2005).

Throughout the flare we applied the multibeam technique described in Costa et al. (1995) and Giménez de Castro et al. (1999), where the signals measured in the three $212-\mathrm{GHz}$ beams $(2,3$, and 4$)$ are used to determine the three unknowns: source intensity and $x, y$ position with time. The positions derived using this method from 17:16:10 through 17:32:51 UT are plotted in Figure 1 as black crosses and are mainly within beam 3. The relative uncertainty in the positions is $5-10$ arcsec and the absolute uncertainty in position is about 30 arcsec (represented by the cross in the upper left corner of the figure). The source positions moved north from 17:16:30 UT to 17:17:10 UT during which the 212-GHz flux density rose to its peak and then moved south later in the flare. If we assume that the $405-\mathrm{GHz}$ source is located at the same place as the $212-\mathrm{GHz}$ centroid emission, we can correct the $405-\mathrm{GHz}$ signal for the Gaussian attenuation caused by an elliptical beam shape of $2 \times 4$ arcmin FWHP. The flux densities at 212 and $405 \mathrm{GHz}$ are calculated by adopting aperture efficiencies of 0.2 and 0.1 , respectively.

The contours of RHESSI hard X-ray images at two different energies are overlaid in Figure 1 for two time intervals. The red contours represent the $100-300 \mathrm{keV}$ emission from 


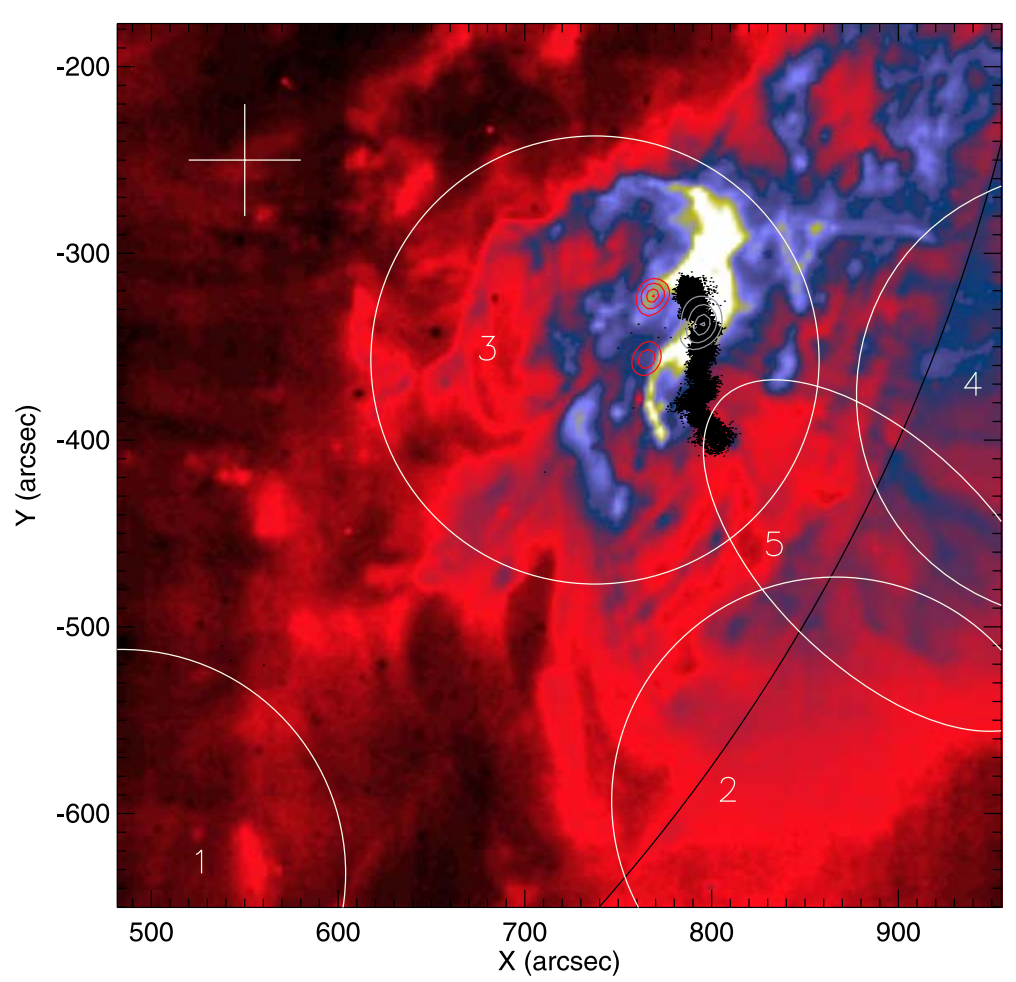

Figure 1 EIT image at 17:12:10 UT with the superimposed multiple beams of the SST at $212 \mathrm{GHz}$ (beams 1 through 4) and $405 \mathrm{GHz}$ (beam 5). The black points indicate the centroid positions of the submillimeter emission. RHESSI 100 - 300 keV sources from 17:16:30 to 17:17:30 UT (red contours) and 12-25 keV from 17:21:30 to 17:22:30 UT (gray contours) are also shown.

17:16:30 to 17:17:30 UT; these agree with the published ones from 200 to $300 \mathrm{keV}$ obtained by Hurford et al. (2006) from 17:14:20 to 17:27:20 UT. The gray contours for $12-25 \mathrm{keV}$ $\mathrm{X}$ rays later in the flare at 17:21:30 - 17:22:30 UT appear to be associated with a bright UV loop. The locations of the submillimeter source shown by the black crosses are consistent with the location of the X-ray sources; however, because of the large absolute uncertainty in radio positions we cannot determine whether the submillimeter source is located at the footpoints or looptop.

\subsection{Comparison of Time Profiles}

The time profiles of the 2 November 2003 flare in microwaves at 11.2 and $18 \mathrm{GHz}$, in the submillimeter at 212 and $405 \mathrm{GHz}$, in soft $X$ rays, from electron bremsstrahlung at 50, 100, and $800 \mathrm{keV}$, and from nuclear de-excitation and annihilation lines are plotted in Figure 2. Two impulsive peaks can be seen in microwaves and hard $X$ rays prior to 17:17:30 UT. Two similar peaks are also seen at 212 and $405 \mathrm{GHz}$; however, they appear to be delayed with respect to the microwaves and $\mathrm{X}$ rays. After that time the radio and $\mathrm{X}$-ray fluxes generally fell over the next 6 minutes with the exception of a broad maximum visible at $\sim 17: 19$ UT in the 212-GHz emission. The fact that this later submillimeter emission was not detected at higher frequency suggests that it originates outside the smaller $405-\mathrm{GHz}$ beam. The nuclear 


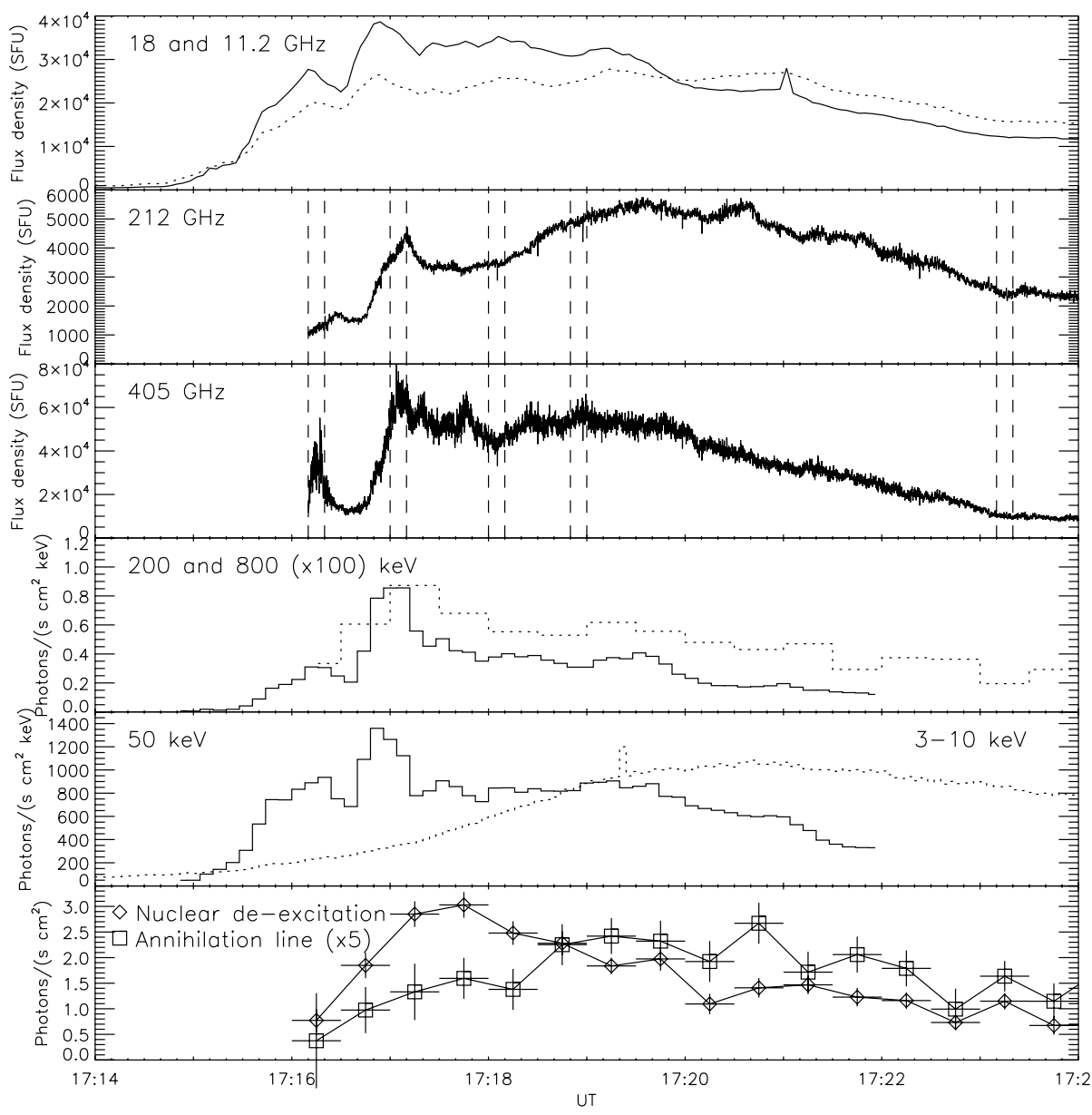

Figure 2 Temporal evolution of the emission at different wavelengths of the 2 November 2003 flare. From bottom to top: nuclear de-excitation (diamonds) and positron 511-keV annihilation (multiplied by five, squares) lines from RHESSI; soft X rays (dotted line), 50-keV and 200- and 800-veV (dotted line, multiplied by 100) hard X rays from RHESSI; 405-and 212-GHz emission from SST; and OVSA flux density at 11.2 (dotted line) and $18 \mathrm{GHz}$.

de-excitation line time profile, which reflects the interaction profile of flare-accelerated ions at energies above $5 \mathrm{MeV} \mathrm{nucl}^{-1}$, peaks later owing to increased ion transport times and also exhibits a long decay. The positron-electron annihilation-line flux peaks even later owing in part to the few-hundred-second decay times of the positron-decay radioactive nuclei.

We have used a cross-correlation study to quantify our visual inspection of the time delays between the impulsive peaks observed in the radio and $\mathrm{X}$ rays. There are no significant delays detected between the peaks of the 11- and $18-\mathrm{GHz}$ emissions and 50-keV X rays, suggesting that $\gtrsim 100 \mathrm{keV}$ electrons are responsible for both microwave and hard X-ray emissions. In contrast both the 212- and 405-GHz emissions exhibit an $\sim 15-20$ s delay from the microwaves and hard $X$ rays. There appears to be a similar long delay $(\sim 20-30 \mathrm{~s})$ between the $800-\mathrm{keV}$ time profile and those measured in the microwaves and hard $\mathrm{X}$ rays. 
This suggests that $\gtrsim 1 \mathrm{MeV}$ electrons produced with a 20-s delay from those at lower energy may be responsible for both the $\mathrm{THz}$ radiation and $>500 \mathrm{keV}$ bremsstrahlung.

Intense submillimeter pulses lasting less than a second were also observed in this flare, similar to what was observed in other large flares (Raulin et al., 2003). Figure 3 displays time profiles of the 212- and 405-GHz emissions for five intervals between the dashed lines in Figure 2. The pulses at both frequencies coincide in time and are more intense at $405 \mathrm{GHz}$, sometimes with flux densities larger than $10^{4} \mathrm{SFU}$ especially during the two main impulsive peaks. The average duration of these pulses is on the order of a few tenths of a second. As the flare evolves, the frequency of occurrence of these pulses decreases, ceasing completely during the decay of the flare as shown in the bottom panel of Figure 3. Unfortunately, it is not possible to search for theses pulses either in the microwave data because of the poor time resolution (4 s) or in the $800-\mathrm{keV}$ bremsstrahlung because of the lack of sufficient statistics.

\subsection{Radio Spectra}

We have constructed flux density spectra from microwave to submillimeter frequencies for the whole duration of the flare. Snapshots of the radio spectrum can be seen in Figure 4 for selected time intervals. During the first two impulsive peaks at 17:16:15 and 17:17:06 UT, the microwave spectra were increasing up to $18 \mathrm{GHz}$, the highest available microwave frequency; therefore we can determine neither the turnover frequency nor the optically thin spectral slope. At later times, the turnover frequency ranges between 11.2 and $18 \mathrm{GHz}$ but we still are unable to determine the optically thin spectral slope. Although the $212-\mathrm{GHz}$ flux density could be an extension of the microwave spectrum, the $405-\mathrm{GHz}$ flux density definitely cannot be. The 212- and 405-GHz flux densities appear to form a new spectral component still rising up to $405 \mathrm{GHz}$, similar to the ones found in the 4 November 2003 flare (Kaufmann et al., 2004) and late in the 28 October 2003 solar flare (Lüthi, Lüdi, and Magun, 2004). The microwave and submillimeter spectra averaged over the time interval from 17:16 to 17:20 UT are shown in the bottom panel of Figure 5.

\section{Origin of the Submillimeter Radiation}

In this section we discuss whether the rising submillimeter spectrum shown in Figure 4 can be explained by optically thick sources such as thermal bremsstrahlung and gyrosynchrotron emission from nonthermal electrons and positrons. We note that the shape of the submillimeter spectrum between 212 and $405 \mathrm{GHz}$ is primarily dependent on the optically thick nature of the source and is only weakly dependent on the emission model. Only with higher frequency measurements can we use the spectral shape to determine the emission mechanism. However, we can use RHESSI observations to constrain the spectra and intensities of flare-produced electrons and positrons and determine whether these fluxes are capable of explaining the bright $\mathrm{THz}$ component.

\subsection{Gyrosynchrotron Radiation from Nonthermal Electrons}

We wish to determine whether we can explain the submillimeter spectral component by gyrosynchrotron emission from flare-accelerated electrons. This same mechanism was proposed by Kaufmann and Raulin (2006) to explain the increasing spectral component also observed on 4 November 2003. 

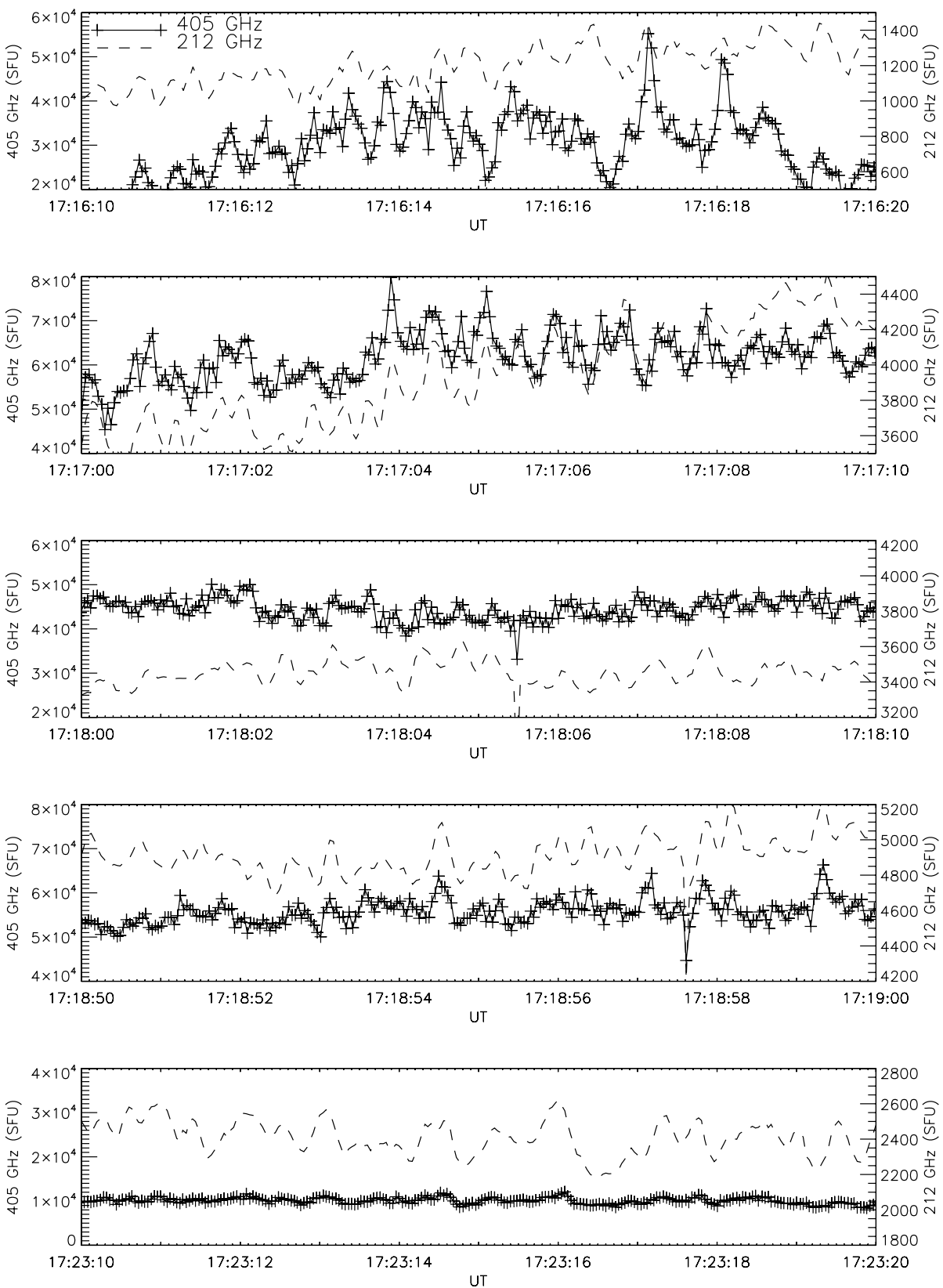

Figure 3 Blowups of selected time intervals, shown between dashed lines in the $212-\mathrm{GHz}$ (dashed) and $405-\mathrm{GHz}$ (crosses) emission of the previous figure. The intense subsecond pulses during the two main impulsive peaks are clearly seen in the top two panels. 

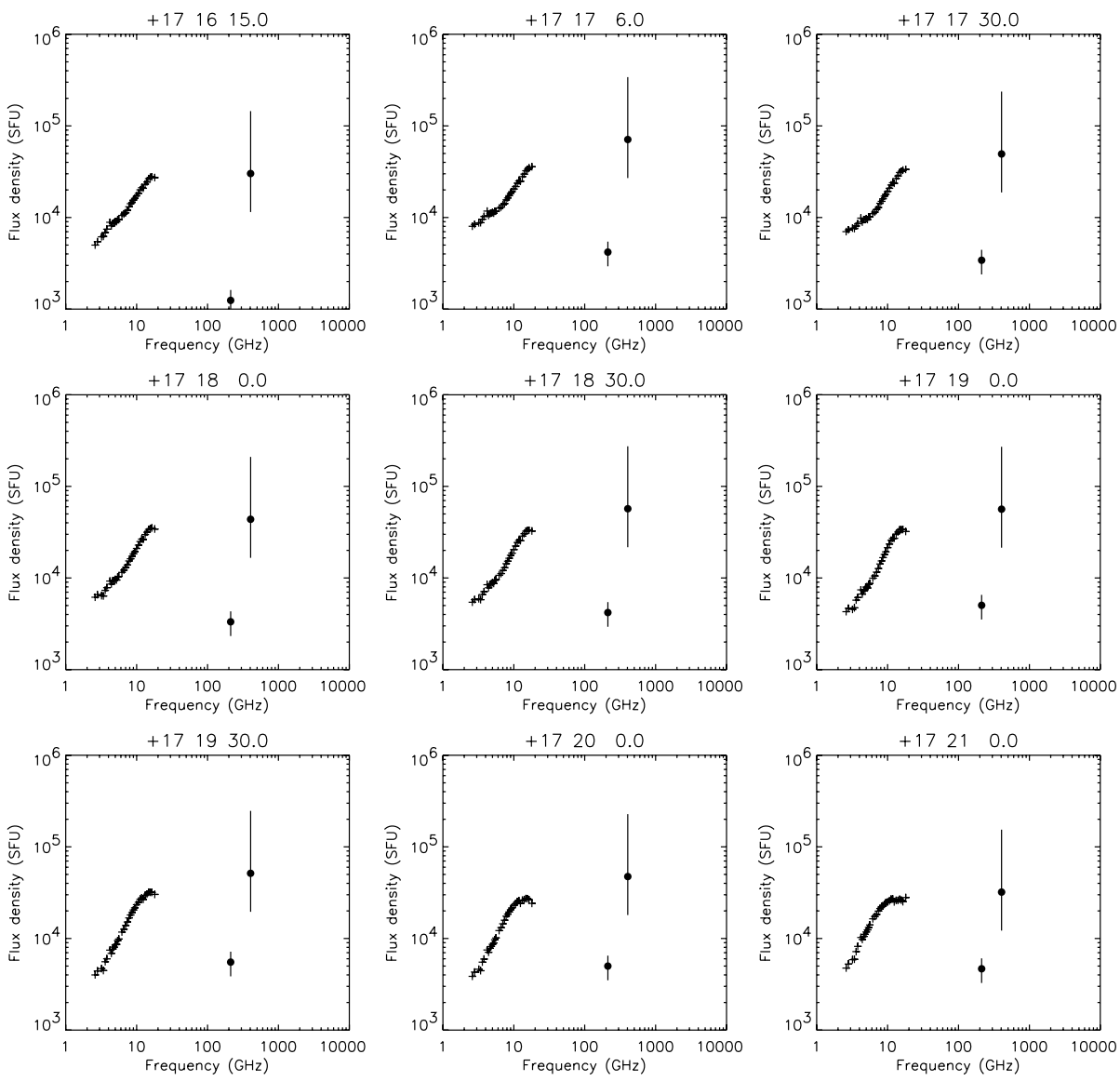

Figure 4 Radio spectra showing the microwave (crosses) and the submillimeter (circles) flux densities at selected time intervals during the 2 November 2003 flare. The first two panels show the spectrum during the two impulsive peaks.

The energy spectrum of electrons that interact in the solar atmosphere can be derived from the bremsstrahlung observed by RHESSI from 17:16 to 17:20 UT, the same interval used in obtaining the radio spectrum shown in the bottom panel of Figure 5 . We fit the RHESSI spectral data from 310 to $16200 \mathrm{keV}$ with a solar photon model that included nuclear-line and bremsstrahlung components. The bremsstrahlung was best modeled with a broken power law having the following parameters: flux at the normalization $310-\mathrm{keV}$ energy of $0.09 \pm 0.005$ photons $\mathrm{cm}^{-2} \mathrm{~s}^{-1} \mathrm{keV}^{-1}$; break energy of $705 \pm 45 \mathrm{keV}$; and power-law indices of $3.05 \pm 0.1$ and $2.15 \pm 0.05$ below and above the break energy, respectively. From a study of the X-ray emission observed in RHESSI's front detector segments we find that the spectrum appears to extend down to about $50 \mathrm{keV}$ without a significant change in spectral index. We then estimated the accelerated electron spectrum from 0.05 to $20 \mathrm{MeV}$ by varying its parameters and using an electron-to-thick-target bremsstrahlung code developed by Holman (2003) until we obtained a good agreement with the RHESSI-derived bremsstrahlung spectrum. Our studies yield a broken power-law spectrum containing at total of $\sim 5 \times 10^{35}$ electrons with indices $\sim 4.15$ and 3.15 below and above a break energy of $\sim 2.8 \mathrm{MeV}$, re- 

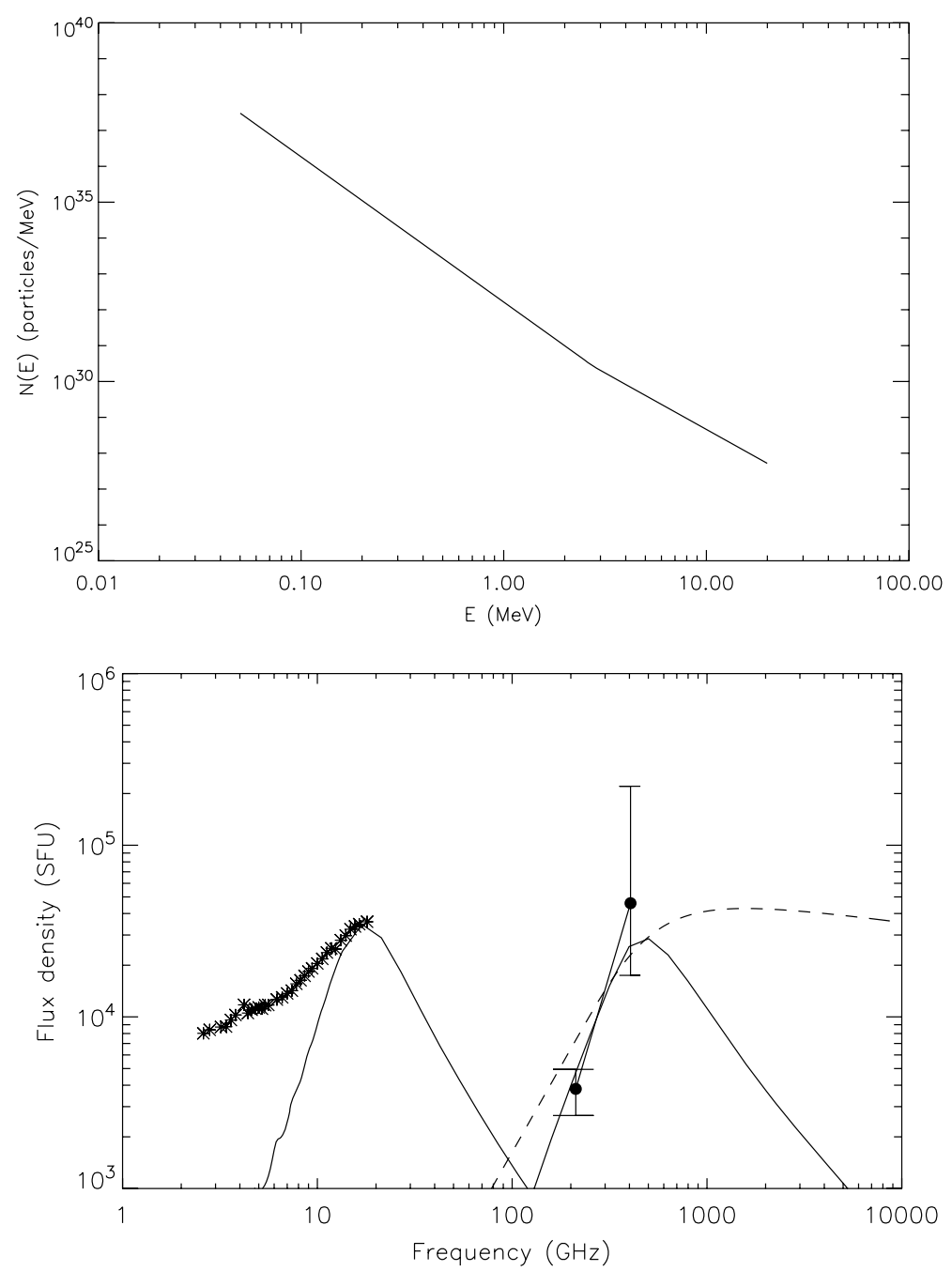

Figure 5 Top panel: Particle energy distribution spectrum for accelerated electron obtained from RHESSI data. Bottom panel: Integrated radio spectra from 17:16 to 17:20 UT; the crosses represent the OVRO microwave emission whereas the filled circles are the submillimeter waves from SST. The dashed curve represents the modeled bremsstrahlung from thermal electrons, whereas the black lines represent the gyrosynchrotron emission from the nonthermal electrons for two different sources (see text).

spectively. This value corresponds to the time-integrated number of electrons present in this flare during the period cited.

We then used this derived electron spectrum to calculate the gyrosynchrotron emission at both microwave and submillimeter wavelengths using a modification of the code developed by Ramaty (Ramaty, 1969; Ramaty et al., 1994; Simões and Costa, 2006) for isotropic electrons having multiple power-law spectra. The additional parameters required by this gyrosynchrotron model are the source size, the magnetic field intensity, and the angle between the line of sight and magnetic field. We used the electron energy spectrum derived from the RHESSI bremsstrahlung observations: a broken power-law between $50 \mathrm{keV}$ and 
$20 \mathrm{MeV}$ containing a total of $5 \times 10^{35}$ electrons. This spectrum is shown in the top panel of Figure 5. We used the flare longitude $56^{\circ}$ as the angle between the line of sight under the assumption that the magnetic loops were perpendicular to the solar surface. With the relatively small number of electrons inferred from the bremsstrahlung, the magnetic field needs to attain values approaching the maximum observed in active regions (Livingston $e t$ al., 2006) (i.e., $4500 \mathrm{G}$ ), while the source size is approximated by a sphere of $\sim 0.5$ arcsec in radius. The modeled gyrosynchrotron emission is plotted as the black curve in the bottom panel of Figure 5. For this small source size, the density of nonthermal electrons is $1.7 \times 10^{12} \mathrm{~cm}^{-3}$. (This is a lower limit to the source density, however, because the emission at frequencies below $405 \mathrm{GHz}$ is optically thick for densities above this value.) Relatively high densities of nonthermal electrons have also been found previously (Ramaty et al., 1994; Raulin et al., 2004). More specifically, (White et al., 2003) found $10^{10} \mathrm{~cm}^{-3}$ above $20 \mathrm{keV}$ at the peak of the 23 July 2002 flare; however this value is $\sim 100$ times lower than required here. In Section 4 we offer plausible scenarios that require less extreme magnetic fields and nonthermal electron densities.

We can also fit the microwave spectrum for the same number $\left(5 \times 10^{35}\right)$ and spectrum of accelerated electrons for a 50" source diameter in a 480-G magnetic field region. This fit is shown as the solid black curve in the bottom panel of Figure 5. Unfortunately, because the microwave emission is optically thick we cannot make an estimative of the electron spectral index.

From this analysis it appears that the microwave and submillimeter emissions originate in two distinctly different environments. The high magnetic field strengths and small source sizes inferred for the submillimeter emission suggests a location relatively low in the solar atmosphere in compact high-density regions perhaps close to the footpoints whereas the lower field strength and larger source size required by the microwave emission reflect lower densities higher in the atmosphere. This model is consistent with our temporal studies indicating close correlation between microwave and $50-\mathrm{keV}$ emissions time profiles and a common delay of $\sim 20 \mathrm{~s}$ observed in the submillimeter and $>800 \mathrm{keV}$ bremsstrahlung owing to the latter's production lower in the solar atmosphere.

\subsection{Bremsstrahlung from Thermal Electrons}

In this section we study the conditions under which bremsstrahlung from thermal electrons can account for the submillimeter observations. Because only the optically thick part of this component is observed, it is not possible to estimate a unique value for the temperature, density, and source size. In addition, we cannot estimate the source size in this flare as was done with the 4 November 2003 flare (Kaufmann and Raulin, 2006) because the source was not located near the intersection of the three $212-\mathrm{GHz}$ beams. We can set some constraints on the parameters if we assume a frequency where the emission becomes optically thin. In the calculations that follow we assume that this occurs at $1 \mathrm{THz}$ and that the source is a cylinder given by its diameter and length. We can then determine the source diameter as a function of the source temperature for a given flux density measured at a specific frequency (Dulk, 1985).

The bottom panel of Figure 6 displays the relationship between source diameter and temperature for the flux density of $4200 \mathrm{SFU}$ at $212 \mathrm{GHz}$ measured near the peak of the impulsive emission at 17:17:06 UT. We see that sources with diameters $<130$ arcsec would require temperatures in excess of $10^{5} \mathrm{~K}$. Similarly, we can estimate the source density as a function of the temperature. This relationship is plotted in the top panel of Figure 6 for three values of source length $\left(10^{8}, 10^{9}\right.$, and $\left.10^{10} \mathrm{~cm}\right)\left(\right.$ Dulk, 1985). In this case, a $2 \times 10^{5} \mathrm{~K}$ 
Figure 6 Source size, or diameter (bottom), and density (top) as a function of temperature needed to fit the submillimeter spectrum as emission from thermal electrons. The density of the cylindrical source is parameterized by its length $L$. These calculations were performed by assuming that the source becomes optically thin at $1000 \mathrm{GHz}$ and by using the 212-GHz flux density at the time of the peak, 17:17:06 UT.

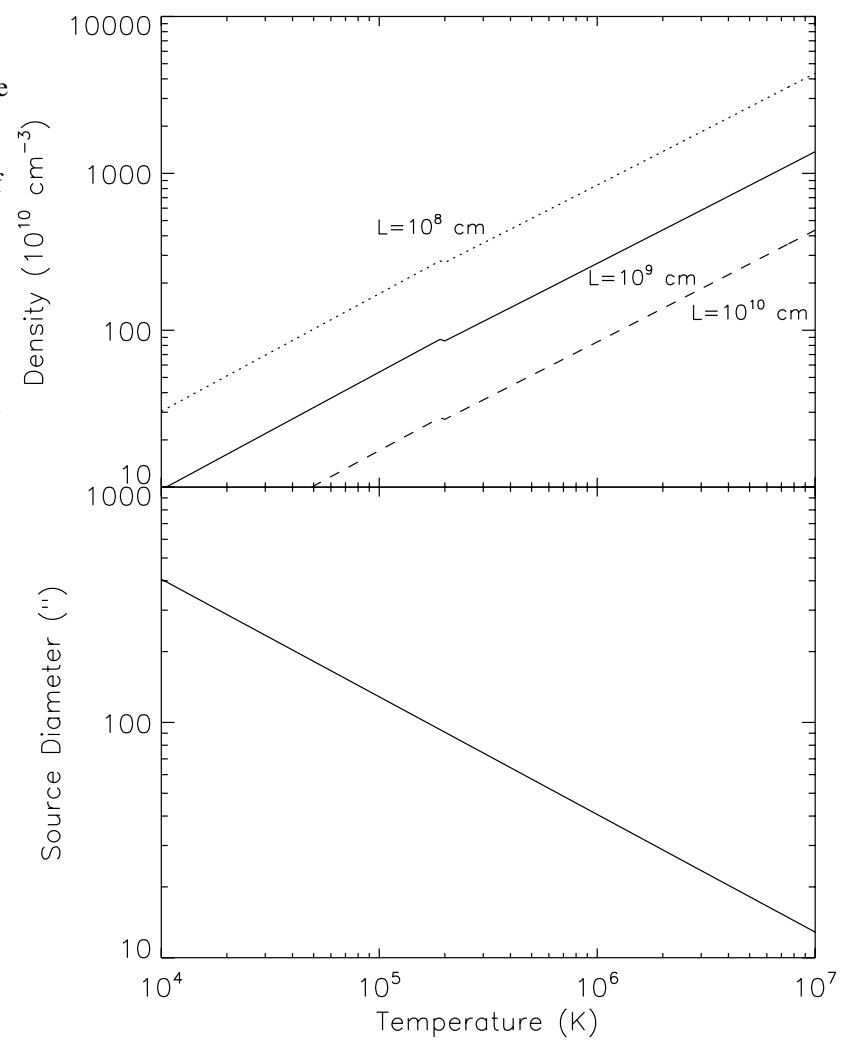

source with $L=10^{9} \mathrm{~cm}$ would have a density on the order of $5 \times 10^{11} \mathrm{~cm}^{-3}$. The free-free emission spectrum fit to the 212- and $405-\mathrm{GHz}$ data for a source with these parameters and diameter of $120^{\prime \prime}$ is shown as the dashed curve in the bottom panel of Figure 5. Such a large source diameter needed to explain the submillimeter observations cannot be ruled out as it is consistent with ultraviolet images obtained at $195 \AA$ by EIT and TRACE later in the flare at 17:36 UT. As previously mentioned, however, the optically thick nature of the source primarily determines the spectral shape.

A thermal bremsstrahlung origin appears to have at least two additional difficulties. A thermal bremsstrahlung origin faces difficulty in explaining both the good temporal agreement between the impulsive submillimeter and hard X-ray fluxes and the subsecond pulses, where the transport of energy may be due to conduction processes. One would have to assume that energy loss from nonthermal particles created the high temperatures in compact sources on the scale of the hard X-ray footpoints. But this would require that the source diameter be $\leq 20$ arcsec. From Figure 6 we see that the temperatures would exceed $10^{6} \mathrm{~K}$ and require very high densities, larger than $10^{13} \mathrm{~cm}^{-3}$, for $L=10^{9} \mathrm{~cm}$. Such a high-density source at the temperatures of millions of kelvins would produce a soft X-ray flux much greater than observed. Using equations in Thomas et al. (1985) for a source at $T=10^{7} \mathrm{~K}$, with density of $n=1.4 \times 10^{13} \mathrm{~cm}^{-3}$ and a radius of $20^{\prime \prime}$ we estimate an X-ray flux equivalent to an X800 class flare. Similar conclusions were obtained for the 4 November 2003 solar event (Kaufmann and Raulin, 2006).

The thermal power radiated during the 2 November 2003 flare inferred from ultraviolet observations by Raymond et al. (2007) is also much too low to explain the thermal 
bremsstrahlung origin for the submillimeter component. We estimate the total thermal energy inferred from the submillimeter observations using $E=3 N K_{\mathrm{B}} T$, where $N$ is the total number of particles, $T$ is the temperature, and $K_{\mathrm{B}}$ is the Boltzmann constant. Using the values computed in Figure 6, we obtain $2.5 \times 10^{30} \mathrm{erg}^{-1} \mathrm{~s}$ for the cooler extended source $\left(T=2 \times 10^{5} \mathrm{~K}, n=5 \times 10^{11} \mathrm{~cm}^{-3}\right.$, diameter of $120^{\prime \prime}$, and length of $\left.10^{9} \mathrm{~cm}\right)$ and $9.6 \times 10^{31} \mathrm{erg}^{-1} \mathrm{~s}$ for the hotter more compact source $\left(T=10^{7} \mathrm{~K}, n=1.4 \times 10^{13} \mathrm{~cm}^{-3}\right.$, diameter of $20^{\prime \prime}$, and length of $10^{9} \mathrm{~cm}$ ). These thermal powers are 100 to 3000 times larger than the values for $L_{\text {therm }}$ listed in Table 3 of Raymond et al. (2007).

\subsection{Gyrosynchrotron Emission from Positrons}

Finally, we consider whether the spectrum and number of positrons estimated from the RHESSI observations can account for the observed submillimeter emission through gyrosynchrotron radiation. We know that positrons were produced in the flare because RHESSI detected a fluence of $\sim 220$ photons $\mathrm{cm}^{2}$ (Share et al., 2004) in the $511-\mathrm{keV}$ annihilation line. Almost all of the 511-keV line radiation must have come from delayed decay of radioactive beta emitters and not from positive-pion decays because (1) we do not find evidence for pion-decay radiation in our fits to the RHESSI spectrum up to $16 \mathrm{MeV}$ and (2) the 511$\mathrm{keV}$ time profile is markedly delayed in Figure 2 and does not follow the same profile as the nuclear de-excitation lines that is expected if the line came from pions decaying in $\ll 1$ second.

If we assume that each positron produced by radioactive decay produces two $511-\mathrm{keV}$ photons and that there is no attenuation in the overlying atmosphere then the measured annihilation line fluence requires a total number of $3 \times 10^{29}$ positrons. To estimate the energy spectrum of these positrons, we consider only the decay from ${ }^{11} \mathrm{C}$ and ${ }^{15} \mathrm{O}$ and assume that each nucleus contributes half of the total number of positrons. The synchrotron emission was estimated as in Section 3.1 using a modified version of the Ramaty code. The resulting flux density that matches the submillimeter emission would be produced by $10^{33}$ positrons in a 0.8 arcsec source in a region of $4500 \mathrm{G}$. This number of positrons required to fit the submillimeter flux density is $\sim 3000$ more than that estimated from the annihilation-line fluence.

The other possibility is that the submillimeter emission arises from higher energy positrons produced by the decay of pions. As previously mentioned, the RHESSI fits to energies up to $16 \mathrm{MeV}$ only yielded an upper limit on the $\gamma$-ray flux from pion decay. This limit is 0.05 photons $\mathrm{cm}^{-2} \mathrm{~s}^{-1}$ from 17:16 to 17:20 UT, which indicates the presence of fewer than $2 \times 10^{26}$ positrons. We used the positron spectrum from pion decay plotted in Figure 16 of Murphy, Dermer, and Ramaty (1987) and then again estimated the gyrosynchrotron/synchrotron emission using the modified Ramaty code. To fit the amplitude and optically thick shape of the submillimeter data points required the presence of $\sim 2 \times 10^{5}$ more high-energy positrons than inferred from the RHESSI observations, a flux density of $4 \times 10^{31}$ positrons from a source size of $0.1 \mathrm{arcsec}$, a $56^{\prime \prime}$ angle between line of sight and magnetic field, and a magnetic field of $4500 \mathrm{G}$.

\section{Summary and Conclusions}

The X8.7 GOES flare on 2 November 2003 was observed both in the submillimeter radio band by the SST and at $\gamma$-ray energies by the RHESSI spectrometer, providing the opportunity to test models suggested for the new submillimeter component (Kaufmann et al., 2004). 
The three suggested origins are (1) gyrosynchrotron emission from flare-accelerated electrons (also responsible for the hard X rays and microwave emission), (2) bremsstrahlung from thermal electrons, and (3) gyrosynchrotron emission from positrons.

The best explanation is that the radio emission is produced by the same acceleratedelectron population responsible for the hard $\mathrm{X}$ rays and $\gamma$ rays. We have derived the total number of nonthermal electrons and spectrum from fits to the RHESSI data and find that they can account for the observed submillimeter radiation under certain conditions, even for an isotropic angular distribution. Our conclusion is also supported by the good correlation between time profiles in the submillimeter and high-energy bremsstrahlung and the presence of rapid subsecond variability in the submillimeter emission. In contrast, the number of positrons produced in radioactive decays following nuclear interactions deep in the solar atmosphere were at least a factor of 3000 too small to account for the submillimeter emission. As we discuss later in this section we can also rule out a thermal bremsstrahlung origin based on time variability and energetic considerations.

Our studies suggest that the same flare-accelerated electrons responsible for the bremsstrahlung can account for both the microwave and submillimeter radio emission. The electron energy spectrum derived from the bremsstrahlung radiation is a double power law with spectral indices of $\sim 4.15$ below $\sim 2.8 \mathrm{MeV}$ and $\sim 3.15$ above this energy, with a total of $\sim 5 \times 10^{35}$ electrons between $50 \mathrm{keV}$ and $20 \mathrm{MeV}$. The isotropic gyrosynchrotron model fitted to the radio data has shown that the microwaves and submillimeter-waves appear to come from two different sources, a more extended one (50 arcsec) located near the looptop with magnetic fields of the order of $480 \mathrm{G}$ and a much more compact source $(\sim 1 \mathrm{arcsec})$ buried deep in the atmosphere in a region of much higher magnetic fields (4500 G). X-ray images of the flare show the traditional footpoint sources observed at the higher energies $(100-300 \mathrm{keV})$ found by Hurford et al. (2006) and a later looptop source at $12-25 \mathrm{keV}$ (see Figure 1). The centroid of the 212-GHz emission lies within the same flaring ultraviolet loop but with the 30 -arcsec uncertainty in its absolute position we are unable to determine whether the submillimeter emission arises from the hard X-ray footpoints or looptop.

There is some substantiating evidence for separate origins of the microwave and submillimeter emissions in our temporal studies. We have found that the time profiles of $50-\mathrm{keV}$ hard $\mathrm{X}$ rays and microwaves observed by OVSA are similar, indicating that electrons of a few hundred $\mathrm{keV}$ are responsible both of these emissions. In contrast we have found that the time profiles of the bremsstrahlung radiation $>800 \mathrm{keV}$ and the $>200 \mathrm{GHz}$ component are similar and delayed from the microwaves, suggesting that higher energy electrons accelerated with a delay are responsible for these emissions. Similar delays between hard X rays of different energies were observed during the 14 August 1979 flare (Vilmer, Trotter, and Kane, 1982) for example, the $398-873 \mathrm{keV}$ were delayed by $36 \mathrm{~s}$ from the $24-43 \mathrm{keV}$ emission. The delays in that flare were successfully explained by efficient trapping of the energetic electrons in a magnetic arch. The constant energetic electron spectral index of 3 used in the Vilmer, Trotter, and Kane (1982) model is similar to what we deduce here; however, because the delays we observe are smaller, this would imply a higher ion ambient density region in the case of the 2 November 2003 flare. Another possibility to account for the delays of the higher energy emission is a second-stage acceleration (Frost and Dennis, 1971) of the electrons, where the $>1 \mathrm{MeV}$ electrons would be accelerated later from an original seed population, thus giving rise to the $\sim 20$ seconds delay of the $800-\mathrm{keV}$ and $212-405$ $\mathrm{GHz}$ emissions.

It is a concern that such high magnetic fields and extreme nonthermal electron densities, requiring a small source size, are needed to fit the increasing spectrum at 212 and 405 GHz. The magnetic field needed to fit the submillimeter spectra is approximately 10 times 
stronger than that needed to fit the microwave spectra. Unfortunately, because this active region, AR 10486, was close to the limb on 2 November there is no accurate measurement of the photospheric magnetic field. An MDI magnetogram of this same region on 29 October, near disk center, indicated maximum fields of $1700 \mathrm{G}$. Nevertheless, Livingston et al. (2006) caution us that the MDI magnetograph, as well as others, fails at measuring strong magnetic fields in sunspot umbrae because of scattered light. For instance, their Table III lists a magnetic field of $4200 \mathrm{G}$ for AR 10484 (Mt Wilson sunspot group 31909) on 22 October 2003; however, the maximum field strength given by the MDI magnetogram for this active region on the same day is only about 1730 G. Since region 10486 was more active than 10484 , in fact producing the largest flare on record two days later on 4 November, it probably had a very high magnetic field at the time of the 2 November flare, even higher than $4200 \mathrm{G}$. In fact, Livingston et al. (2006) report that $0.2 \%$ of almost 32000 active regions studied have sunspots with magnetic fields larger than $4000 \mathrm{G}$, even as high as $6100 \mathrm{G}$, the highest magnetic field ever measured.

Our simplifying assumption that electrons were isotropically distributed may contribute to the requirements of high magnetic field strength and subarcsec source size. For the gyrosynchrotron emission from the same number of nonthermal electrons (from RHESSI) to match the submillimeter observations for the same source size (radius of 0.5 arcsec) but with a smaller magnetic field, for example $B=1000 \mathrm{G}$, we need the peak frequency to basically triple from $160 \mathrm{GHz}$ to the observed lower limit of about $500 \mathrm{GHz}$, and the flux density to increase by a factor of 9. Fleishman and Melnikov (2003) have shown that these conditions can be achieved by considering the emission from nonisotropic electron distributions; see, e.g., the intensity plotted in their Figure 13 for the case of high-density plasma. Note, however, that their electron spectrum is much steeper (index $=7$ ) than we have found for the 2 November flare. The authors argue that the increase in intensity and turnover peak frequency are easily achieved for different parameters of a beamlike distribution. Center-tolimb variations in the spectra of high-frequency microwave flares (Silva and Valente, 2002) and $\gamma$-ray bremsstrahlung (Vestrand et al., 1987) suggest strong anisotropies in the parent electron populations.

We also note that we have not considered transport effects in our calculations. Since the gyrosynchrotron emission is occurring at a different location than the bremsstrahlung, the electrons may lose energy before reaching the atmospheric depth where the bremsstrahlung is produced. Therefore the spectra and numbers of electrons producing the observed submillimeter and microwave radiation may be significantly different from those producing the $\mathrm{X}$ and $\gamma$ rays. Thus, it may be reasonable to speculate that the number of electrons available to produce the gyrosynchrotron radiation is higher than that giving rise to the bremsstrahlung (Raulin et al., 1999). Once again this would lower the magnetic field strength required to fit the submillimeter spectra.

Kaufmann and Raulin (2006) investigated the same type of rising submillimeter spectra in another flare and suggested that they arise from synchrotron radiation from beams of ultrarelativistic electrons $(>10 \mathrm{MeV})$ under magnetic fields ranging of the order of $1000 \mathrm{G}$. The beams may be submitted to microbunching instabilities, giving rise, at the same time, to intense broadband coherent synchrotron radiation at microwave wavelengths. Thus this new spectral component, which has only been observed in a few flares, may require the presence of regions with intense magnetic fields.

For completeness, we have also investigated whether thermal bremsstrahlung can account for the rising submillimeter component. This has appeal because of the high brightness expected at submillimeter wavelengths but such emission would typically require temperatures of $4 \times 10^{5} \mathrm{~K}$ over a 50 -arcsec source radius at ambient densities of $10^{11}-10^{12} \mathrm{~cm}^{-3}$. Such 
a source size is consistent with submillimeter measurements of the 28 October 2003 flare; Lüthi, Lüdi, and Magun (2004) found that the source radius varied from about 80 to less than 10 arcsec during the highly impulsive phase and then maintained a radius of $\sim 55$ arcsec after 11:07 UT. We were not able to measure the size of the submillimeter source in the 2 November 2003 flare because it was not located near the center of the three beams of the telescope as was the source of the 4 November 2003 flare. There is evidence from $\gamma$-ray observations for thermally broadened annihilation-line radiation in some flares, suggesting temperatures in excess of $10^{5} \mathrm{~K}$ (Share et al., 2004). We studied the $511-\mathrm{keV}$ line width in the 2 November flare using both RHESSI front and rear detectors. We find that the line can be well fit by positrons annihilating deep within the chromosphere under relatively quiet conditions and does not require such elevated temperatures. We obtain an upper limit of $\sim 10^{5} \mathrm{~K}$ on the temperature where annihilations took place. If such a region had produced the submillimeter radiation it would have had to have a radius in excess of $\sim 120$ arcsec (from Figure 6).

There are other difficulties for the thermal bremsstrahlung origin. The close association of the high-energy $X$ rays and submillimeter time profiles would require that high-energy electrons heated the medium in the compact hard X-ray footpoints in Figure 1. To produce the submillimeter emission by thermal bremsstrahlung in such a compact 20 -arcsec source, a temperature of $10^{7} \mathrm{~K}$ and density of order $10^{12}-10^{14} \mathrm{~cm}^{-3}$ would be required. However, this would result in too much soft X-ray emission (about 100 times more than ever observed). Moreover, the estimated thermal power from the submillimeter observations is three orders of magnitude larger than that inferred from ultraviolet observations of the 2 November flare (Raymond et al., 2007).

The novel characteristic of this flare at radio wavelengths is its spectral component increasing at $>200 \mathrm{GHz}$ frequencies, distinct from the well-known broadband spectral component observed at microwaves. Based on our study of the 2 November 2003 flare, we find that the most likely source is gyrosynchrotron radiation from nonthermal electrons, but even this explanation may require adopting high local magnetic fields, very large densities of accelerated electrons, and very small source sizes, considering that the same number of electrons produce the $\mathrm{X}$ rays and submillimeter and microwave radiation. Observations at even higher frequencies $(\mathrm{THz})$ are desired to better constraint the cause of this new spectral component.

Acknowledgements The authors are grateful for fruitful discussions with T.S. Bastian, D.E. Gary, S.M. White, and J.C. Raymond. We acknowledge partial support from the Brazilian agencies FAPESP (Grant No. 99/06126-7), CNPq, and the Argentinean agency CONICET. G.S. and R.M. acknowledge support from NASA Grant NNG05GP76G and DPR NNG05HL38I. The authors thank the OVSA for making the microwave data available on their homepage and the RHESSI team.

\section{References}

Costa, J.E.R., Correia, E., Kaufmann, P., Magun, A., Herrmann, R.: 1995, Solar Phys. 159, 157.

Dulk, G.A.: 1985, Ann. Rev. Astron. Astrophys. 23, 169.

Fleishman, G.D., Melnikov, V.F.: 2003, Astrophys. J. 587, 823.

Frost, K.J., Dennis, B.R.: 1971, Astrophys. J. 165, 655.

Giménez de Castro, C.G., Raulin, J.-P., Makhmutov, V.S., Kaufmann, P., Costa, J.E.R.: 1999, Astron. Astrophys. Suppl. Ser. 140, 373.

Holman, G.D.: 2003, Astrophys. J. 586, 606.

Hurford, G.J., Krucker, S., Lin, R.P., Schwartz, R.A., Share, F.H., Smith, D.M.: 2006, Astrophys. J. 644 , L93.

Kaufmann, P., Raulin, J.: 2006, Phys. Plasma 13, 070701.

Kaufmann, P., Correia, E., Costa, J.E.R., Zodi Vaz, A.M.: 1986, Astron. Astrophys. 157, 11. 
Kaufmann, P., Costa, J.E.R., Giménez de Castro, C.G., Hadano, Y.R., Kingsley, J.S., Kingsley, R.K., et al.: 2001, In: Pinho, J.J., Cavalcante, G.P.S., Oliveira, A.H. (eds.) Proc. 2001 SBMO/IEEE MTT-S International Microwave and Optoeletronics Conference, 439.

Kaufmann, P., Raulin, J.-P., Melo, A.M., Correia, E., Costa, J.E.R., Giménez de Castro, C.G., et al.: 2002, Astrophys. J. 574, 1059.

Kaufmann, P., Raulin, J.-P., Giménez de Castro, C.G., Levato, H., Gary, D.E., Costa, J.E.R., et al.: 2004, Astrophys. J. 603, L121.

Lin, R.P., Dennis, B.R., Hurford, G.J., Smith, D.M., Zehnder, A., Harvey, P.R., et al.: 2002, Solar Phys. 210 , 3.

Livingston, W., Harvey, J.W., Malanushenko, O.V., Webster, L.: 2006, Solar Phys. 239, 41.

Lüthi, T., Lüdi, A., Magun, A.: 2004, Astron. Astrophys. 420, 361.

Lüthi, T., Magun, A., Miller, M.: 2004, Astron. Astrophys. 415, 1123.

Murphy, R.J., Dermer, C.D., Ramaty, R.: 1987, Astrophys. J. Suppl. Ser. 63, 721.

Ohki, K., Hudson, H.S.: 1975, Solar Phys. 43, 405.

Ramaty, R.: 1969, Astrophys. J. 158, 753.

Ramaty, R., Schwartz, R.A., Enome, S., Nakajima, H.: 1994, Astrophys. J. 436, 941.

Raulin, J.-P., White, S.M., Kundu, M.R., Silva, A.V.R., Shibasaki, K.: 1999, Astrophys. J. 522, 547.

Raulin, J.-P., Kaufmann, P., Giménez de Castro, C.G., Pacini, A.A., Makhmutov, V.S., Levato, H., et al.: 2003, Astrophys. J. 592, 580.

Raulin, J.P., Makhmutov, V.S., Kaufmann, P., Pacini, A.A., Lüthi, T., Hudson, H.S., et al.: 2004, Solar Phys. 223, 181.

Raymond, J.C., Holman, G., Ciaravella, A., Panasyuk, A., Ko, Y.-K., Kohl, J.: 2007, Astrophys. J. 659, 750.

Sakai, J.I., Nagasugi, Y.: 2007, Astron. Astrophys. 470, 1117.

Sakai, J.I., Nagasugi, Y., Saito, S., Kaufmann, P.: 2006, Astron. Astrophys. 457, 313.

Share, G.H., Murphy, R.J., Smith, D.M., Schwartz, R.A., Lin, R.P.: 2004, Astrophys. J. 615, L169.

Silva, A.V.R., Laganá, T.F., Giménez de Castro, C.G., Kaufmann, P., Costa, J.E.R., Levato, H., et al.: 2005, Solar Phys. 227, 265.

Silva, A.V.R., Valente, M.M.: 2002, Solar Phys. 206, 177.

Simões, P.J.A., Costa, J.E.R.: 2006, Astron. Astrophys. 453, 729.

Stein, W.A., Ney, E.P.: 1963, J. Geophys. Res. 68, 65.

Thomas, R.J., Crannell, C.J., Starr, R.: 1985, Solar Phys. 95, 323.

Trottet, G.: 2006, In: Fang, C., Schmieder, B., Ding, N.U.P. (eds.), Third French-Chinese Meeting on Solar Physics, 82 .

Trottet, G., Raulin, J.-P., Kaufmann, P., Siarkowski, M., Klein, K.-L., Gary, D.E.: 2002, Astron. Astrophys. 381, 694.

Trottet, G., Lüthi, T., Myagkova, I.N., Dauphin, C., Magun, A., Vilmer, N., et al.: 2004, In: 35th COSPAR Scientific Assembly, 2234.

Vestrand, W.T., Forrest, D.J., Chupp, E.L., Rieger, E., Share, G.H.: 1987, Astrophys. J. 322, 1010.

Vilmer, N., Trotter, G., Kane, S.R.: 1982, Astron. Astrophys. 108, 306.

White, S.M., Krucker, S., Shibasaki, K., Yokoyama, T., Shimojo, M., Kundu, M.R.: 2003, Astrophys. J. 595, L111. 